\title{
Development of Non-invasive Electroencephalography Technique in Animal Model
}

\author{
Vasudeva Murthy Sindgi ${ }^{1, \star}$, Mounika Basani ${ }^{1}$, Pragna Choudary ${ }^{1}$, Kashinath Molugu ${ }^{1}$, Ramesh \\ Malothu' ${ }^{2}$
}

${ }^{1}$ Jayamukhi College of Pharmacy, Narsampet, Warangal, Telangana, INDIA.

${ }^{2}$ School of Biotechnology IST, JNTUK - Kakinada, Andhra Pradesh, INDIA.

\begin{abstract}
Aim: This paper describes about the new non-invasive method to measure brain waves from the scalp of the rats. The method allows to record good quality Electroencephalogram (EEG) from freely moving rats in the cage. Methods: This experiment targeting rodents used simple adhesive patch containing conductive gel placed on the scalp of the rats has a potential to measure the brain waves without any surgical intervention. Stable electrode gel shown to have good conductivity and strongly bonds to the skin of the animal and is well suited for recording from awaken and freely moving rats. We used two channel data acquisition system to convert analogue to digital signals of the brain waves. This study conducted to record the accurate electroencephalograph signals, a helpful tool for the translational research. The major advantages of the non-invasive EEG recording in contrast to the invasive method we presented here an efficacious, quick and efficient technique for the placement of the EEG leads in rats. Results: Quality EEG data obtained using two channel data acquisition system and is used for analysis. Reliable estimation of the electrical activity of the brain from conscious rat can be made. In conventional procedures electrodes are implanted in the skull with an additional stressful procedure. Conclusion: The results provided evidence that this method can be used as an alternative to the conventional recording of the electroencephalogram. This tool definitely a valuable tool in basic research, neurodegenerative and epilepsy research.
\end{abstract}

Key words: Electroencephalogram, Fast Fourier Transform, Stereotaxis equipment, Frequency bands, Conductive gel, Absolute Power.

\section{INTRODUCTION}

The present invention provides a disposable lead relates to an Electroencephalogram (EEG) component device for recording Rat / rodent brain physiological changes in terms of electrical signals in the form of EEG. Non-invasive method is used to investigate brain function of human beings have been used in research laboratories for many decades; however, their reputation has increased in recent years given the ease of use and broad application. Such methods have proved a valuable in improving our knowledge about numerous areas of basic brain function. Many non-invasive techniques applied to patient groups to identify pathological mechanisms, ${ }^{1}$ but decisively a new role has found for some as biomark- ers of disease. ${ }^{2}$ Neurodegenerative disease is fast becoming one of the principal medical problems in the first world. The recent Neuroprotective drugs offer the potential to slow the progression of some diseases. For avian behaviour and physiological research was carried on laying hens but in this case electrodes are inserted subcutaneously and called it as non-invasive method. ${ }^{3}$ EEG recorded in dairy cow for brain activity, eye movements and muscle activity recorded using electromyography. Non-invasive method using adhesive electrodes secured to the shaved skin of the cow. EEG analyzed using digital recording and Power spectrum was calculated using Fast Fourier Transform (FFT). This method established
Submission Date: 22-05-2019; Revision Date: 24-06-2019; Accepted Date: 06-09-2019

DOI: 10.5530/ijper.53.4s.157 Correspondence:

Dr. Vasudeva Murthy, Jayamukhi College of Pharmacy, Narsampet, Warangal 506 332, Telangana, INDIA. Phone: +918143635527 E-mail: sgvmurthy@gmail. com

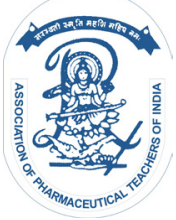

www.ijper.org 
and possible to distinguish different vigilance states in freely moving cows. ${ }^{4}$

In another technique needle electrode filled with conductive gel placed on the head of the rat and EEG recorded in freely moving rats. Brain electrical activity was extracted using non-invasive approach. ${ }^{5}$ Electroencephalogram is in use to ascertain the actions of the drugs acting on the nervous system ${ }^{6}$ and intracranial electrodes placed using stereotaxic equipment and involving surgical procedure. ${ }^{7}$ There is currently an immense need to enhance the clinical diagnostic range with reliable, robust and specific biomarkers of neurodegenerative disease.

However, careful and rigorous studies to authenticate the uses of non-invasive techniques during last 80 years basic science exposed several methods to study functioning of the brain. These methods include invasive approaches, such as electrical recording of impulse activity of single and multi-neurons, recording local field potentials and intracranial Event-related Potentials (ERPs), as well as polarographic recordings of brain tissue oxygen. ${ }^{8}$ This research paper describes about the technique which allow non-invasive recording of faithful EEG signal from rat. Electrodes placed on the surface of the scalp, which doesn't need brain surgery. Conventional method uses intracranial electrode implantation and requires technical surgical skills, induces post operative trauma and stress. ${ }^{9}$ The traditional technique used to study the effects of drugs induced changes in EEG spectral content. eg. Systemic administration of benzodiazepines, such as diazepam produces sedative effect and recording the signature effect of the increase in the beta band can be illustrated. ${ }^{10}$

Object of the current invention is to determine the EEG spectral bands frequency to determine absolute and relative power of EEG to characterize wave patterns for the purposes of treatment or in diagnosing neuronal diseases or to monitor the effects of the various therapeutic agents or phytochemicals using noninvasive method and can be used during the preclinical trials apart from the alertness or cognitive engagement or to investigate sleep disorder.

\section{MATERIALS AND METHODS}

After the approval of the Institutional Animals Ethics Committee (01/JCP/IAEC/2016) the research was conducted on the animals. The present invention provides a disposable EEG leads, a component of Electroencephalogram (EEG) device for recording Rat / rodent brain activity and to note the physiological changes in terms of electrical signals in the form of
EEG. The disposable EEG leads placed on the surface of the rat's cleanly shaven head or using any depilatory agent. The disposable EEG leads placed in three areas, on the cortex of the left parietal region is positive and negative EEG lead was placed on right parietal region and neutral or ground lead was placed on nasal bone area of the rat. By this method various parameters of the brain physiology recorded in the form of various bands viz. gamma, delta, theta, beta and alpha. The spectral content of the rat EEG was determined by the brain activity. Brief description of drawings for a better understanding of the present invention mentioned in drawings of the EEG leads and reference to its parts shown in the Figure 1. Referring to Figures, EEG leads consists of skin contacting surface material such as stainless steel, silver, or nickel. The scalp contacting surface of the EEG lead coated with a layer of conducting material or gel layer that is about $3-4 \mathrm{~mm}$ diameter and thickness of approximately $1 \mathrm{~mm}$ being preferred. Backing is preferably made up of a vinyl foam plastic with adhesive coating. The EEG lead is square in shape with approximately 5 sq.mm placed on the surface of the rat head.

\section{Detailed description of the invention}

Non-invasive electroencephalogram is a recording of the electrical activity of the brain from the scalp. The waveforms recorded thought to reflect the activity of the surface of the brain, the cortex. This activity influenced by the electrical activity from the brain structures underneath the cortex. The electroencephalogram is cord of the oscillations of brain electric potentials tracing electrodes attached to the scalp. The recorded signals transmitted to an EEG system composed of amplifiers, filters and computer monitor. The brain electrical charge is maintained by billions of neurons. As the wave of ions arrives at the electrodes on the scalp, they can push or pull electrons on the metal on the electrodes. Known that metal conducts the push and pull of electrons easily, the difference in drive or draw voltages between any two electrodes can be measured by a voltmeter. Recording these voltages over time gives us the EEG. Brain patterns form wave shapes that are commonly sinusoidal when acquired from the animal. Generally, they are measured from peak to peak and normally range from 0.5 to $100 \mathrm{pV}$ in amplitude, which is about 100 times lesser than Electrocardiogram (ECG) signals. In the present studies by the way of Fourier transform power spectrum from the raw EEG signal is derived. The EEG signal, additionally categorized into, depending on the frequency ranges. EEG activity can be broken down into 5 distinct frequency bands: gamma 
( $40 \mathrm{~Hz}$ to $100 \mathrm{~Hz}$ ), delta activity $<4 \mathrm{~Hz}$, theta activity - $4 \mathrm{~Hz}-8 \mathrm{~Hz}$, beta activity $-12 \mathrm{~Hz}$ to $40 \mathrm{~Hz}$ and alpha activity $-8 \mathrm{~Hz}-13 \mathrm{~Hz}$. Method developed, recorded and measured in microvolts $(\mu \mathrm{V})$ with the main frequencies of interest up to approximately $100 \mathrm{Hertz}(\mathrm{Hz})$. The electrical signal obtained from EEG measurements analyzed with spectral analysis methods using Biopac with AcqKnowledge version 4.4 software. In spectral analysis, the EEG signal is seen as the sum of multiple sine waves each with its own frequency and amplitude. Through the Fast Fourier Transform (FFT) the EEG signal is decomposed into multiple sign waves of different frequencies and the amplitude of each frequency can be expressed as power $\left(\mu \mathrm{V}^{2}\right)$.

\section{Placement of electrodes}

Electrodes were prepared by extracting three very thin insulated thin wires, the inner copper wire is exposed and is inserted through a $3 \mathrm{sq} \mathrm{mm}$ of adhesive plaster. To the tip of all three electrodes, electrode gel obtained from the commercially available ECG leads manufactured by $3 \mathrm{M}$, India, this electrode gel use potassium chloride for ion conductivity and are glycerin based, information about the contents of the gel obtained via email from the office of the $3 \mathrm{M}$ Infection-Prevention Division, 3M Center, St. Paul, MN 55144. USA. Conductive gel (2-3\%) contains: water (80-90\%), propylene glycol (10-20\%), potassium chloride $(2-5 \%)$ and hydroxypropyl guar gum (1-3\%). Approx 25mg of conductivity gel used for study and is purchased from commercially available ECG leads (3M TM -India) and extracted gel was applied on to the exposed wire. The rat was held in between the two palms by retracting rats two forelimbs and the animal was made immobile and the entire setup of plaster with electrodes attached to the wire pasted on the surface of the animal scalp which was earlier depilated using a depilatory agent then pasted the electrodes by applying to the contour of the foam plastic a instant glue, a monomer called cyanoacrylate applied to fix firmly to depilated head of the rat. The electrodes were placed on the cortex of the left parietal is positive; negative was placed on right parietal region and neutral or ground was on nasal bone region of the rat.

The data acquisition system using bipolar electrodes with a ground electrode used in EEG recordings. The recording cables were connected to a scalp of the rat and allowed free movement of the animal in the cage and allowed to acclimatize to the new environment for fifteen min before the starting of the recording. Signals were filtered through a high pass $0.5 \mathrm{~Hz}$ and low pass $100 \mathrm{~Hz}$. Digitization (sampling rate: 200 samples /sec) and data recording were done using Biopac USA. EEG recording inspected visually and artefact-containing EEG was discarded before analysis.

\section{RESULTS}

EEG recording on the day of the experiment, the animals were adapted to the experiment at conditions for about 15 min before starting the recording. Water and food was withheld during experiment. EEG recordings were carried out in the time of $9.00 \mathrm{am}$ to $2.00 \mathrm{pm}$. EEGs were recorded using Biopac, USA and processed EEG signals were connected to a microcomputer system coupled with a fast fourier transformation (FFT). For 12 wistar rats of either sex weighing 180-200g used for the study. For this experiment, the drug-free baseline (control) activity was recorded for $30 \mathrm{~min}$. In case, for the evaluation of the any drug, a required dose administered to the rat was given orally or parental route and EEG recording was commenced and recorded for $30 \mathrm{~min}$ or recorded based on the individual drug pharmacokinetics profile. Power spectrum analysis was used for EEG data analysis. In brief, this involves separating the EEG signal into fundamental frequencies and determining the amount of frequency in the record. The data are then displayed as power as a function of frequency. The sweeps of $30 \mathrm{sec}$ activity were averaged as spectra of power density $\left(\mu \mathrm{V}^{2} / \mathrm{Hz}\right)$ on the monitor and stored in a file. Data in successive time-blocks of $3 \mathrm{sec}$ epoch of 5 averages were summed up again and averaged to obtain log values. The EEG spectra of each was calculated into percentage of the baseline activity and used for further analysis.

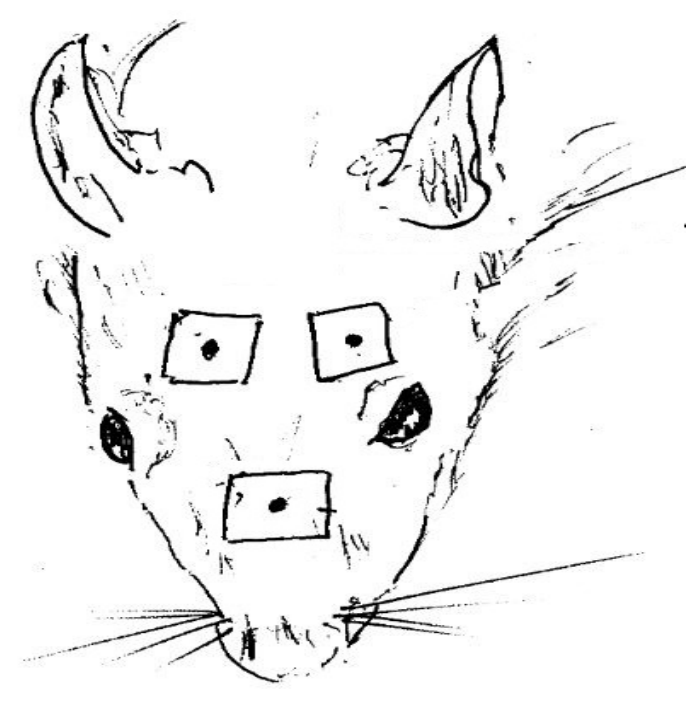

Figure 1: Placement of EEG leads on the surface of the rat scalp 


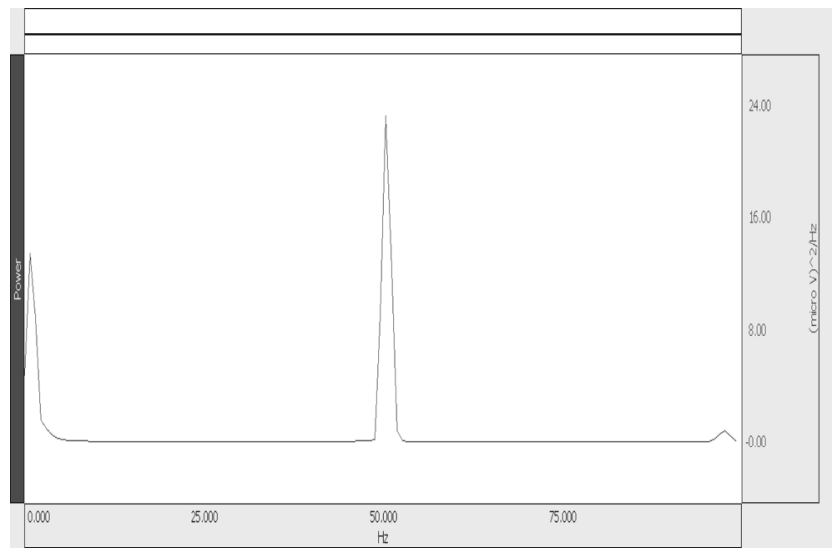

Figure 2: The spectrum identifies electrical frequency peak at $50 \mathrm{~Hz}$ due to electrical interferences.

Artefacts those are sufficiently short or long to have probably been caused by non-physiological variables, such as noise or signal artefacts, one such example of artefacts due electric current/volts appeared at 50 Volts (Figure 2).

Figure 3 represents presence of overall view of beta, alpha, theta and delta and gamma bands during the EEG recordings in Selective Serotonin Reuptake Inhibitors (SSRI) treated rat with venlafaxine.

\section{Calculation}

The spectra for artefact-free epochs were averaged by taking 3 average values for a $10 \mathrm{sec}$ epoch. From the averaged spectral power values, Absolute Power (AP) and Relative Powers (RP) were calculated.

\section{DISCUSSION}

The invention relates to modelling and designing of EEG leads to suit the rat and other non rodent animals for detecting and recording the complex patterns of neural activity occurring within fractions of a second after a stimulus or after drug administration. The device comprises foam plastic material and at the centre of the EEG lead a metal, usually a copper/ silver, a good conductor of the signals generated in the brain. The change in EEG parameters in some diseases and disorder of the central nervous system can be recorded. A digital EEG system converts the waveform into a series of numerical values. This process known as Analogueto-Digital conversion (ADC). The values can be stored in the computer memory, manipulated and then redisplayed as waveforms on a computer screen. The invention extends a method of non-invasive monitoring of rodent and non rodent animals and this method specifically described to obtain the faithful EEG signals.

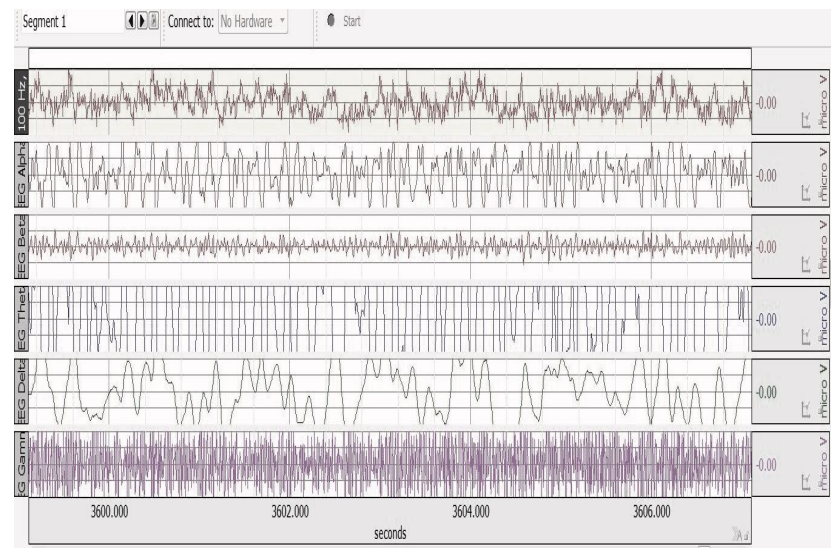

Figure 3: EEG recording representing the presence of overall view of beta, alpha, theta, delta and gamma bands.

\section{CONCLUSION}

The method describing non-invasive recording of the EEG experiment have all the characteristics of recording faithful brain waves. This experiment having the potential to measure the electrical activity of the rat brain and can be done without any surgical intervention.

\section{ACKNOWLEDGEMENT}

SVM is grateful to AICTE, New Delhi for receiving Grant under Research Promotion Scheme (RPS) (Ref. No. 8-125/RIFD/RPS/POLICY-3/2013-14) to carry out project work on 'Development of Non-invasive EEG Technique in Animal Model'

\section{CONFLICT OF INTEREST}

The authors declare that there are no conflicts of interest.

\section{ABBREVIATIONS}

EEG: electroencephalogram; FFT: Fast Fourier Transform; ERPs: Event-Related Potentials; $\mu \mathrm{V}$ : Microvolts; ECG: Electrocardiogram; AP: Absolute Power; RP: Relative power; ADC: Analogue-to-Digital conversion.

\section{REFERENCES}

1. 1. Hughes SW, Crunelli V. Thalamic Mechanisms of EEG Alpha Rhythms and Their Pathological Implications. Neuroscientist. 2005;11(4):357-72.

2. 2. Al-Qazzaz NK, Ali SHBM, Ahmad SA, Chellappan K, Islam MS, Escudero J. Role of EEG as Biomarker in the Early Detection and Classification of Dementia. Sci World J. 2014;2014:1-16.

3. 3. Eberle KN, Martin MP, Shah S, Malheiros RD, Livingston KA, Anderson KE. A novel non- invasive method for evaluating electroencephalograms on laying hens. Poultry Sci. 2018;97(3):860-4.

4. 4. Ternman E, Hänninen L, Pastell M, Agenäs S, Nielsen PP. Sleep in dairy cows recorded with a non-invasive EEG technique. Appl Anim Behav Sci. 2012;140(1-2):25-32. 
5. 5. Ferrari R, Arce AIC, Melo MPD, Costa EJX. Noninvasive method to assess the electrical brain activity from rats. Ciênc Rural. 2013;43(10):1838-42.

6. 6. Inmsen $H$, Schywalsky $M$, Plettke $R$, Priller $M$, Walz F, Schwilden $\mathrm{H}$. Concentration-Effect Relations, Prediction Probabilities $(\mathrm{Pk})$ and Signal-to-noise Ratios of Different Electroencephalographic Parameters during Administration of Desflurane, Isoflurane and Sevoflurane in Rats. Anesthesiology. 2008;108(2):276-85.

7. 7. Dimpfel W. Pharmacological modulation of cholinergic brain activity and its reflection in special EEG frequency ranges from various brain areas in the freely moving rat (Tele-Stereo-EEG). Eur Neuropsychopharmacol. 2005;15(6):673-82.

8. 8. Bartlett K, Saka M, Jones M. Polarographic Electrode Measures of Cerebral Tissue Oxygenation: Implications for Functional Brain Imaging. Sensors. 2008;8(12):7649-70.

9. 9. Turner PV, Kloeze H, Dam A, Ward D, Leung N, Brown EEL, et al. Mass depopulation of laying hens in whole barns with liquid carbon dioxide: Evaluation of welfare impact. Poultry Sci. 2012;91(7):1558-68.

10. 10. Vanlier $\mathrm{H}$. Effects of diazepam and zolpidem on EEG beta frequencies are behavior-specific in rats. Neuropharmacology. 2004;47(2):163-74.

\section{PICTORIAL ABSTRACT}

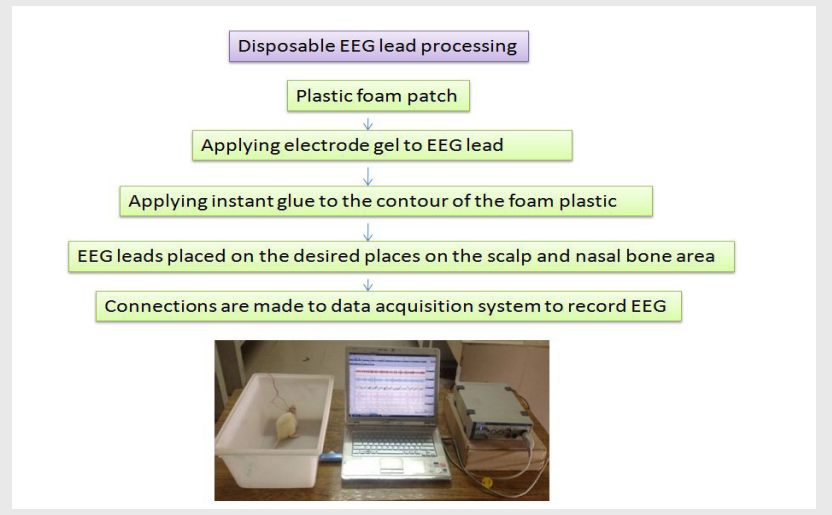

\section{SUMMARY}

The new method of recording of the EEG assembly allows us to record faithful electroencephalogram. Prepared EEG lead has a significantly higher success rate. The prepared EEG patch is economical and made easily obtainable materials and minimum skills are essential to record brain waves.

\section{About Authors}

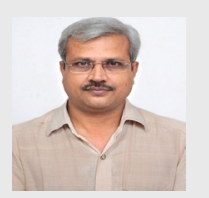

Vasudeva Murthy Sindgi, obtained Ph.D. in Pharmaceutical Sciences from Kakatiya University, Warangal, Telangana, India, 2001-2005. B.Pharm and M.Pharm from Gulbarga University. Gulbarga. Currently working at Jayamukhi College of Pharmacy, Narsampet, Telangana. Awarded FIP (International Pharmaceutical Foundation) Fellowship in the year 2003 from the FIP Foundation for Education and Research, The Hague, Netherlands, on "Host defense factors in nasal fluid from leprosy patients" and visited University of Central Florida, Orlando. FL, USA.

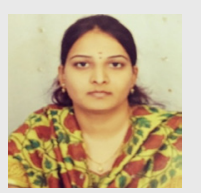

Mounika Basani, a research scholar from Jawaharlal Nehru Technological University, Kakinada after obtaining B.Pharm and M.Pharm from Kakatiya Univeristiy, Warangal. Worked as a Assistant Professor in the Department of Pharmaceutics, in Jayamukhi College of Pharmacy, Narsampet, Warangal Rural. Supervised M.Pharm students for their project work in the area of Novel Drug Delivery System. She has many publications in the area of Pharmaceutical formulations. She is a life member of APTI.

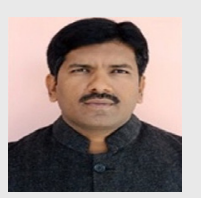

Dr.Ramesh Malothu, B.Tech, M.Tech, PhD. Assistant Professor, JNTU, Kakinada, Andhra Pradesh. He pursued Master's Degree from West Bengal University of Technology, Kolkata sponsored by Department of Biotechnology (DBT), Govt. of India, New Delhi. He did a Doctoral Degree in the field of Enzyme Engineering and molecular Biology from JNT University, Kakinada. Dr. Ramesh $\mathrm{M}$ so far has successfully guided $22 \mathrm{M}$.Tech and M.Pharmacy students in the fields of Enzyme Engineering, rDNA Technology and Immunotechnology, etc. Dr. Ramesh Malothu has received prestigious award from Department of Science and Technology (DST) New Delhi as a Fast Track Young Scientist Award in 2013. Dr.Ramesh Malothu has filed 5 Indian Patents to his credit.

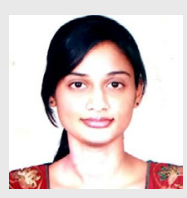

Pragna Choudary, is M.Pharm (Pharmacology) from Jayamukhi College of Pharmacy, affiliated to Kakatiya University, Warangal.

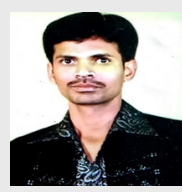

Kashinath Molugu, is M.Pharm (Pharmacology) from Jayamukhi College of Pharmacy, affiliated to Kakatiya University, Warangal

Cite this article: Murthy VS, Basani M, Choudary P, Molugu K, Malothu R. Development of Non-invasive Electroencephalography Technique in Animal Model. Indian $\mathrm{J}$ of Pharmaceutical Education and Research. $2019 ; 53(4 s): s 619-s 623$. 